\title{
Evaluation of Oilseeds for Biodiesel Production
}

\author{
Cinthia da Silva Carreiro da Luz ${ }^{1}$, Fernando B. Mainier ${ }^{2, *}$, Luciane Pimentel Costa Monteiro \\ ${ }^{1}$ Instituto Federal do Rio de Janeiro, Niterói, RJ, Brazil \\ ${ }^{2}$ Universidade Federal Fluminense, Niterói, RJ, Brazil
}

\begin{abstract}
Biofuels are less polluting alternatives because they are not fossil fuels. In Brazil, the biodiesel program aims to incorporate raw vegetable material into conventional diesel oil (2 to 5\%) or even replace it entirely as a fuel for adapted diesel engines. As Brazil is one of the largest producers of soybeans in the world, soybeans are the oleaginous plant most used for biodiesel production in Brazil, even if they don't constitute the best option in relation to the yield of oil produced. The oil content of soybeans is about $19 \%$, while canola and sunflower feature, respectively, 38 and $42 \%$ oil. The productivity of oil (in terms of $\mathrm{kg} / \mathrm{ha}$ ) of both canola and sunflower also surpasses soybeans. In addition to the three already mentioned oils (canola, sunflower and soya), coconut and corn oils were studied, totaling five oils. Transesterification was used with ethanol via basic catalysis using sodium hydroxide catalyst. The reaction time was 30 minutes, with the original oils heated to $50^{\circ} \mathrm{C}$; however, the reactions were carried out at room temperature. The synthesis with coconut oil had the lowest yield; the others (canola, sunflower, maize and soya) achieved yields on the order of $70 \%$.
\end{abstract}

Keywords Biodiesel, Ethanol, Fuel, Soybeans, Transesterification, Synthesis

\section{Introduction}

There is constant concern in the modern world about the deep dependence of and competition by industrialized countries for precious and finite fossil hydrocarbons, whether in the form of oil or natural gas. These are considered the foundation of the contemporary economy, since three-quarters of global energy comes from fossil fuels. Is valid to say that the great demand for fuel is forcing the depletion of primary sources, driving the need for replacement energy sources, especially when they are associated with the new environmental vectors.

The high levels of contaminants from these sources, such as carbon dioxide $\left(\mathrm{CO}_{2}\right)$, sulfur oxides $\left(\mathrm{SO}_{2}, \mathrm{SO}_{3}\right)$ and nitrogen oxides $\left(\mathrm{NO}_{\mathrm{x}}\right)$, methane $\left(\mathrm{CH}_{4}\right)$ and associated sources of industrial raw materials end up producing harmful air pollution and, as a consequence, directly or indirectly, wind up contaminating water sources, natural reserves and food sources, causing immense damage to humanity $[1,2]$.

Alternative renewable sources have been presented and discussed in the world aiming to replace, in part, the dependence on fossil fuels.

In Brazil, the biodiesel national program aims to incorporate raw vegetable material into conventional diesel oil ( 2 to $5 \%)$ or even replace it entirely as a fuel for adapted diesel engines. It is important to note that, as it is derived from vegetable oils, biodiesel has reduced sulfur levels and

* Corresponding author:

fmainier@uol.com.br (Fernando B. Mainier)

Published online at http://journal.sapub.org/ajee

Copyright (C) 2015 Scientific \& Academic Publishing. All Rights Reserved does not generate pollutants during its industrial production. This characteristic is an important factor fostering its adoption, due to great world pressure for the use of clean energy. In this regard, it is worth mentioning that this procedure displays, in addition to economic and ecological benefits, an important role in social inclusion. In this case energy production is associated with rural producers. This incentive encourages farmers to remain in the fields and reduces urban overpopulation $[3,4]$.

The use of vegetable oil as a fuel for diesel engines has already occurred once in the past, when Rudolf Diesel himself conducted tests with a diesel engine based on peanut oil around 1893. In the 20th century, however, diesel engines were adapted for the combustion of petroleum products, which were relatively more affordable and abundant compared with vegetable oils at that time. Interest was renewed only in 1970, after the first oil shock and the growth of ecological awareness prompted the rediscovery of vegetable oil-based fuels as part of the process of seeking substitutes for hydrocarbon-based fuels [5].

The burning of organic fuel, fossil or not, emits $\mathrm{CO}_{2}$. A renewable fuel from biomass is considered "carbon neutral", since the $\mathrm{CO}_{2}$ emitted was previously consumed by the plant in the course of photosynthesis, which removes $\mathrm{CO}_{2}$ from the atmosphere and releases $\mathrm{O}_{2}$. Fossil fuels, by contrast, are not considered "carbon neutral" because the burning of these causes a considerable increase in $\mathrm{CO}_{2}$ levels in the atmosphere, major urban centers being the places most affected due to their large numbers of combustion-powered cars.

Biodiesel is an efficient, clean, $100 \%$ natural energy fuel 
alternative, offering the same performance and engine durability in addition to being non-flammable, non-toxic, non-aromatic, and biodegradable; furthermore, it does not emit sulfur compounds into the environment [6].

To minimize this strong dependence on fossil fuels, bi-fuel cars, popularly known as flex cars, whose Otto motors work both with alcohol and gasoline, have necessitated some changes to the engines so they can work with both fuels. Gasoline already has such a replacement renewable ethanol. Biodiesel, likewise, presents itself as a renewable substitute for fossil diesel, with the advantage of not requiring major changes in Diesel engines, it being possible to use any proportion of biodiesel/diesel.

The Brazilian model, the development of biofuels contributes to a sustainable vision of his energy policy. Taking into consideration the environmental, social and economic aspects and the contributions of the various sectors that make up society (public and private).

This vision drives process called a "triple bottom line", which gives the model of development of biofuels, the form of a model directed to sustainable development.

Based on these premises of sustainability was created the "Social Fuel Seal", in 2004, granted to biodiesel producer that can promote and support social inclusion of family farmers providing raw material in their small rural properties. Industries who buy from small farmers receive the "Social Fuel Seal".

A comparison between the creation of occupations in agriculture business and in the family farmers shows that in the agriculture business, on average a worker employed for every 100 hectares cultivated; while in the familiar, the relationship is only 5-10 hectares per worker. However, given the diversity of raw materials and the processing is critical the quality criterion.

The main criterion for the quality of biodiesel is the care of an appropriate standard. Generally, the fuel quality can be influenced by several factors, including the quality of the raw material, the composition in fatty acids from vegetable oil or animal fat, the production process, the use of other materials in the production process and subsequent parameters. The presence of water and sediments are considered fundamental for the quality control of biodiesel.

Brazil is a country with great potential for renewable energy production by virtue of possessing advantages in agronomic conditions, since the climate is tropical, with high rates of luminosity and annual average temperatures, water availability and a variety of choices of cultivated oleaginous plants. The most-produced oilseed on national (domestic) soil is soybean, and Brazil is the second largest producer of soybeans in the world, second only to the United States. Brazilian soy production closed the year of 2012 with 66.68 million tons, while the Americans scored 83.17 million tons $[7,8]$.

This article aims to present, at bench level, ester yields produced through reactions with canola oil, coconut oil, sunflower oil, corn oil and soybean oil. We also consider other data in the literature, in terms of the oil content in the seeds and oil productivity per area planted, in order to identify the best options for the production of biodiesel.

\section{Transesterification Reaction}

To transform the oil extracted from of oilseeds into biodiesel, transesterification has to be employed, which consists basically in the removal of a molecule of glycerin from the oil, as illustrated in Figure 1. For this reaction to occur, it is necessary to react the oil with a short chain alcohol such as methanol or ethanol in the presence of a catalyst. After the reaction, two phases are formed: glycerin (more dense) and biodiesel (less dense). Such stages may be separated by decanting or centrifugation.

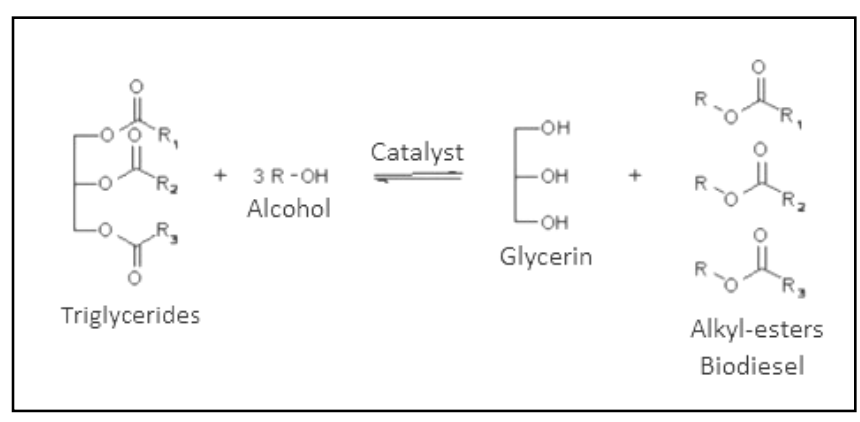

Figure 1. Transesterification reaction

The stoichiometry of the reaction requires 3 moles of alcohol for each mole of triglyceride. As the reaction is reversible, an excess of alcohol will cause the equilibrium to be displaced in favor of the products, increasing the efficiency of esterification. Certain variables can influence the transesterification reaction, such as: purity of reagents, molar ratio of alcohol/oil, reaction temperature, catalyst and agitation.

From the operational point of view, the route using methanol is simpler, with a shorter reaction time, more spontaneous separation of the glycerin and ester phases, and high conversion rates; only a small excess of alcohol is needed. However, methanol is highly toxic and is also derived from non-renewable sources; in addition, Brazil is not self-reliant in its productions. In contrast, ethanol has low toxicity and is produced from renewable sources (agricultural products). In addition, Brazil is the largest producer of ethanol from sugar cane in the world $[9,10]$.

With respect to the type of catalysis, the reaction can occur along a homogeneous catalytic pathway (basic or acid) or a heterogeneous one. In basic homogeneous catalysis, alkali metal hydroxides, carbonates and alkoxides of alkali metals (sodium methoxide, sodium ethoxide, and piperonyl sodium) are usually used. Industrially, this is the most suitable route, because it is faster than homogeneous acid catalysis; in addition, alkali catalysts are easier to manipulate and less corrosive than acid catalysts. This pathway occurs at atmospheric pressure, under mild temperatures and a lower molar ratio of alcohol/oil compared to homogeneous acid catalysis. 
Alkoxides of alkali metals are the most active catalysts, with high yields $(>98 \%)$ and short reaction times $(30$ minutes), even at low concentrations $(0.5$ mole $\%)$. However, they require the absence of water, since this leads to hydrolysis of the ester produced with consequent formation of soap. This saponification reaction reduces the yield of ester and hinders the process of separating the phases [11]. Another feature of the reagents is related to their acidity index, resulting from the presence of free fatty acids in the oil. Such acids will consume the basic catalyst, undermining the development of the reaction.

Homogeneous acid catalysis is more common for esterification reactions. The catalyst used most often in this case is sulfuric acid $\left(\mathrm{H}_{2} \mathrm{SO}_{4}\right)$. This route is mainly used for the catalytic production of biodiesel from waste with high levels of free fatty acids. Acid catalysis has a satisfactory reaction speed for esterification reactions. However, for transesterification reactions, acid catalysis has the disadvantage that it requires a high alcohol: oil molar ratio, requiring high temperatures and long periods of synthesis [12].

Heterogeneous catalysis is an alternative to the problems found in homogeneous catalysis such as: increased number of steps; purification of products; possible corrosion of the reactor vessel (acid catalysis); saponification (basic catalysis); and low activity against alcohols of higher molar mass. In addition, it has advantages: ease of purification of monoesters, allowing the reuse of catalysts, minimizing the generation of effluents and considerably facilitating the recovery and purification of glycerin. The most commonly used catalysts are: zeolites, oxides and inorganic salts, anion exchange resins, organic acids and bases and lamellar materials [13].

\section{Materials and Methods}

The focus of the present study is based on comparing the ester yields from canola, coconut, sunflower, corn and soybean oils using the ethanol process for the catalytic pathway with sodium hydroxide $(\mathrm{NaOH})$ and excess $100 \%$ ethanol.

The laboratory test consisted essentially of heating the oil to a temperature of $50^{\circ} \mathrm{C}$ and then preparing the sodium ethoxide, dissolving it in $\mathrm{NaOH}$ in anhydrous ethyl alcohol in an Erlenmeyer flask with a glass cover in order to prevent contamination with moisture and thus prevent the saponification reaction.

The heated oil and sodium ethoxide were then added to a $400 \mathrm{ml}$ beaker with a magnetic stirrer that maintained constant stirring for exactly 30 minutes. After 30 minutes the reaction mixture was transferred to a decanting funnel and left to rest for 24 hours; afterward, the two phases were separated. The denser phase (glycerin) was discarded and the less dense phase was neutralized and dried with $\mathrm{Na}_{2} \mathrm{SO}_{4}$.

\section{Results and Discussion}

As stated previously, the processing of the oils was the same. All the synthesis presented a slight darkening of the color with soybean oil presenting a different feature. The blackout, for soybeans, at the time of adding the alkoxide, was pretty intense at the beginning. But, over the course of the reaction, the color became less intense until it turned brown. This fact has also been observed and mentioned in surveys conducted by Pelisson [14].

Probably, this change in color is due to the lecithin, chemically named fatty or phospholipid. Lecithin is removed from crude oil in the gum removal process (degumming) and the resulting oil presents an amber color. Different oilseeds will present different levels of phospholipids, but soybeans stand out among the other oilseeds, their fatty content reaching around 3\% in the crude oil. During the process of degumming, only hydratable phospholipids are extracted, the non-hydratable phospholipids remaining, these being the responsible for the brown color when heated [15].

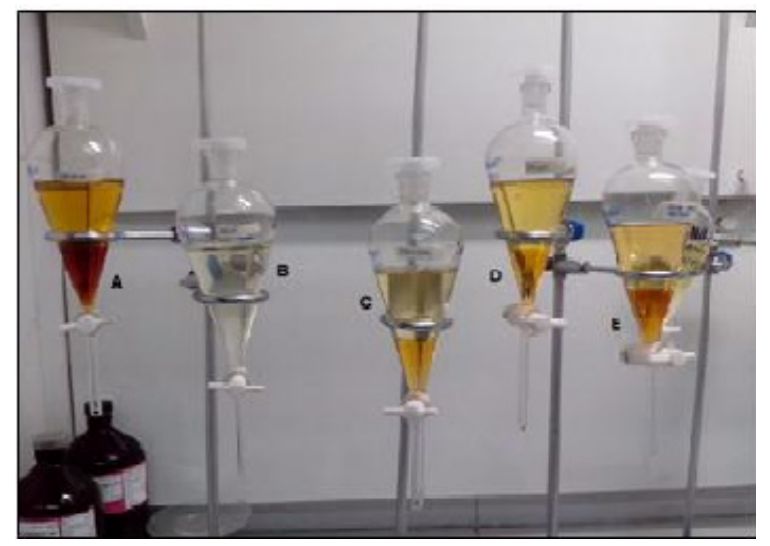

Figure 2. Glycerin-ester phase separation of canola (A), coconut (B), sunflower(C), corn (D) and soybeans (E)

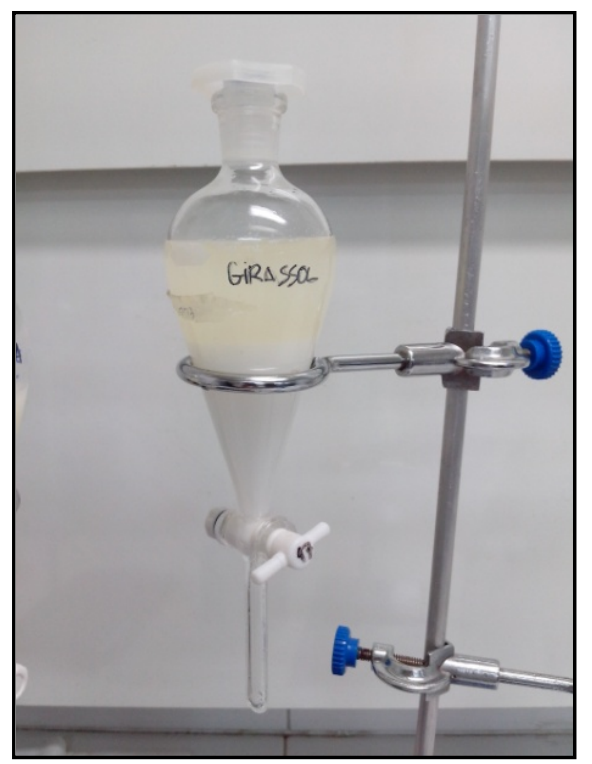

Figure 3. First wash with sunflower oil (C) 
After a period of 24 hours, we noticed the formation of two phases in all syntheses except the coconut. Thus, glycerin was added to the separator funnel containing the synthesis with the coconut oil for easy viewing of the two phases, as illustrated in Figure 2.

The first wash was done with water slightly acidified with hydrochloric acid in order to neutralize the basic catalyst, as well as remove excess alcohol ester phase. In this step, it was noted that soap was formed in the aqueous phase (denser), possibly due to oil that did not react, as illustrated in Figure 3. In this case, the occurrence of the saponification reaction shows interesting to remove the oil that had not yet responded, getting on stage essentially ester biodiesel.

The second wash was performed two or three times with deionized water until the water stayed clear and had a neutral $\mathrm{pH}$. To remove the residual water content from the oily phase, sodium sulfate $\left(\mathrm{Na}_{2} \mathrm{SO}_{4}\right)$ was added and then filtered out. The liquid was then weighed to determine the mass of the ester.

Table 1 presents a comparison between the studied oils (canola, coconut, sunflower, corn and soya) being considered, in addition to the yield in terms of Brazil's agricultural production of each oil.

Table 1. Comparison between the studied oils

\begin{tabular}{|c|c|c|c|}
\hline Type of oil & $\begin{array}{c}\text { Oil yield based } \\
\text { on Brazilian } \\
\text { agricultural } \\
\text { production (t/ha) }\end{array}$ & $\begin{array}{c}\text { Ester yield } \\
(\%)\end{array}$ & $\begin{array}{c}\text { Ester yield based } \\
\text { on Brazilian } \\
\text { agricultural } \\
\text { production (t/ha) }\end{array}$ \\
\hline Canola & $0,5-0,9$ & 72,61 & $0,36-0,65$ \\
\hline Coconut & $0,3-1,4$ & 66,86 & $0,20-0,94$ \\
\hline Sunflower & $0,5-1,5$ & 71,28 & $0,36-1,07$ \\
\hline Corn & $0,18-0,36$ & 71,40 & $0,13-0,26$ \\
\hline Soybeans & $0,2-0,6$ & 72,51 & $0,15-0,44$ \\
\hline
\end{tabular}

Except for the coconut, all other oilseeds had ester yields around $72 \%$; however, both canola and sunflower have high annual yields of oil in terms of tons per hectare and also good yields in ester production, reaching values more than twice the yield with soy oil, which is the currently most widely used oil for biodiesel production in Brazil.

Glycerin, despite being, in principle, undesirable, can be a product with high commercial value, especially in the cosmetics industry, which could bring economic advantages for the production of biodiesel.

With respect to the price of refined oil, it has recently been observed that soy is the cheapest; sunflower and corn oils cost approximately twice as much as canola and soy is slightly more expensive than these two. The price of coconut oil is extremely high, and may be up to 30 -fold greater than that of soybeans, making it uncompetitive in economic terms.

\section{Conclusions}

The exhaustion of fossil fuel reserves is foreseeable, which makes it important to search for alternative fuels, not only because of the issue of shortages, but also the need to reduce pollution and improve air quality in major cities.

Biodiesel has come to be the renewable diesel substitute of choice, just as alcohol is for petrol, soybeans being the most used for biodiesel production in Brazil. The oil content in their grains is $19 \%$; other oilseeds, however, such as canola and sunflower, respectively, feature twice as much or even more oil. In addition, canola and sunflower also outperform soybeans in terms of oil yield per unit area.

It is important to note that all oilseeds study obtained ester yields around $72 \%$ with the exception of coconut.

With the biodiesel industry still in its early stages, perhaps this difference is subtle enough that it will not have a great impact. However, the search for an oilseed crop must include the desirability of a lower demand for land. With the decreasing supply of fossil fuel will come an increase in the consumption of renewable fuel. This will result in increased demand for land for the cultivation of oleaginous plants for biofuel. In the process of expanding agricultural frontiers, one has to think of an oleaginous plant that can produce a large amount of oil in a smaller area of cultivation and will give a good yield of ester. On these issues, both canola and sunflower are not suitable, which makes them good alternatives to soybeans for biodiesel production.

\section{REFERENCES}

[1] S. Nakano, A. Okamura, N. Sakurai, M. Suzuki, Y. Tojo, and N. Yamano, N., "The Measurement of $\mathrm{CO}_{2}$ Embodiments in International Trade: evidence from the harmonized input-output and bilateral trade database", Organization for Economic Co-operation and Development (OECD), February, 2009.

[2] A. Demirbas, "Biodiesel, a realistic fuel alternative for diesel engines", Springer, London, 2008.

[3] R. Bailis, Brazil: Biodiesel. Sustainable Development of Biofuels in Latin America and the Caribbean. Springer New York, pp.103-126, 2014.

[4] J. Goldemberg, and S. T. Coelho, S. T., Bioenergy: how much? Environmental Research Letters, v. 8, n. 3: 031005, 2013.

[5] G. Knothe, Dependence of biodiesel fuel properties on the structure of fatty acid alkyl esters, Fuel processing technology, v. 86, 10: pp. 1059-1070, 2005.

[6] K. Bozbas, Biodiesel as an alternative motor fuel: Production and policies I the Europe Union, Renewable and Sustainable Energy Reviews, pp. 1-12, 2005.

[7] Brazil, 2013. Ministério da Agricultura (Ministry of Agriculture), Statistical yearbook of agroenergy 2012, Brasília, http://www.agricultura.gov.br/, Access in: 12/02/2014, (in Portuguese). 
[8] J. C. Bergmann, D. D. Tupinambá, O. Y. A. Costa, J. R. M. Almeida, C. C. Barreto and B. F. Quirino, Biodiesel production in Brazil and alternative biomass feedstocks. Renewable and Sustainable Energy Reviews, 21: pp. 411-420, 2013.

[9] S. P. Sing, and D. Dipti, Biodiesel production through the use of different sources and characterization of oils and their esters as the substitute of diesel: A review, Renewable and Sustainable Energy Reviews, 14: pp. 200-216, 2010.

[10] P. P. K. G. Costa, Chemical catalysts used in the synthesis of biodiesel. Brasília, DF: Brazil, Embrapa Agroenergia, 2011. (in Portuguese).

[11] U. Schuchardt, R. Sercheli and R. M. Vargas, Transesterification of vegetable oils: a review. J. Braz. Chem. Soc. vol.9, 3 :pp. 199-210, 1998.
[12] C. L. M. Silva, Obtaining ethyl esters from the transesterification of andiroba with ethanol, Dissertation (MSC. in chemistry), Unicamp, São Paulo, Brazil, 2005. (in Portuguese).

[13] C. A. R. Melo Jr, Catalytic and non-catalytic esterification for synthesis of biodiesel in microwave reactor. Dissertation (Msc in Process Engineering), Universidade Tiradentes, Aracaju, SE, Brazil, 2008. (in Portuguese).

[14] L. Pelisson, Biodiesel production by means of pressurized fluids and their characterization using high resolution gas chromatography (HRGC), Doctoral thesis, Universidade de São Paulo (USP), São Carlos, Brazil, 2013. (in Portuguese). 